\title{
Parametric Krylov-based order reduction of aircraft aeroelastic models
} Computational Reduced-Order Models I 


\section{Motivation}

Objective: use the interpolation of reduced order aeroelastic systems to update internal models for control in real time

Computationally demanding processes performed offline: building the library of reduced order models from highly refined nonlinear aircraft systems

Reduced order systems are of an adequate size and accuracy for the interpolation to be performed online at the desired operating condition

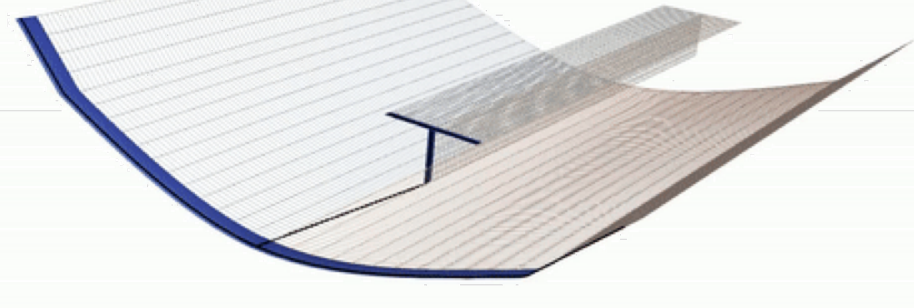

Nonlinear Simulation of a Very flexible aircraft flying through a gust. 
Nonlinear Aeroelastic Modelling 


\section{Nonlinear Aeroelastic Modelling}

SHARPy: Simulation of High Aspect Ratio aeroplanes and wind turbines in Python ${ }^{1}$

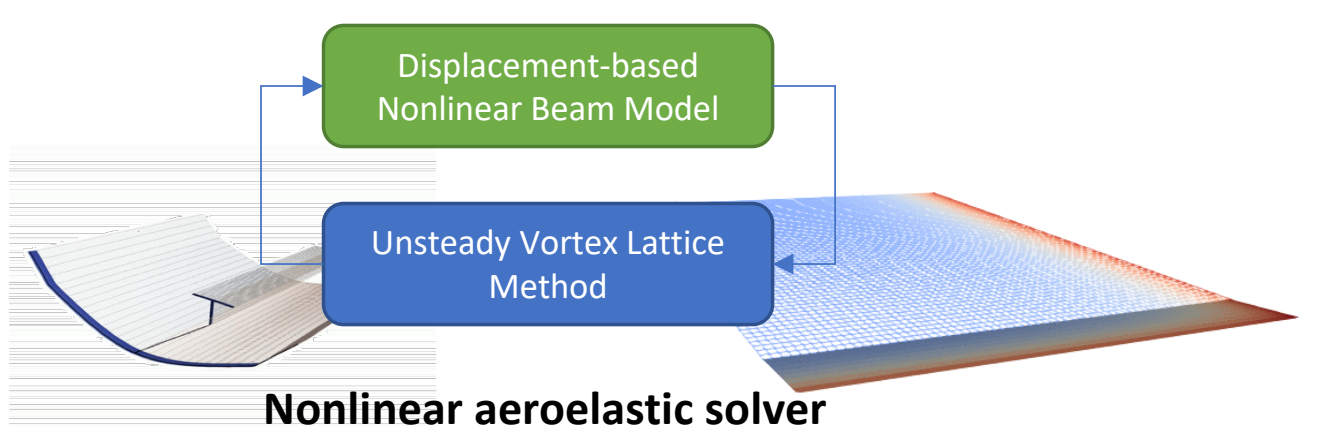




\section{Nonlinear Aeroelastic Modelling}

SHARPy: Simulation of High Aspect Ratio aeroplanes and wind turbines in Python ${ }^{1}$

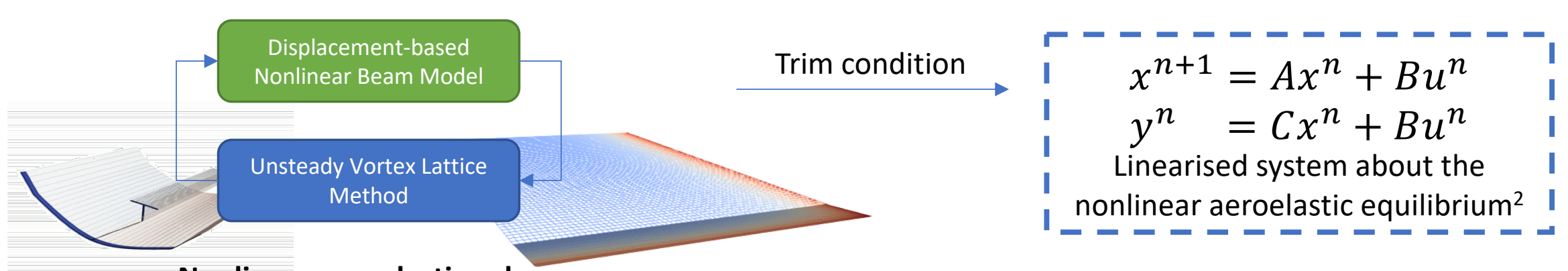

Nonlinear aeroelastic solver 


\section{Nonlinear Aeroelastic Modelling}

SHARPy: Simulation of High Aspect Ratio aeroplanes and wind turbines in Python ${ }^{1}$

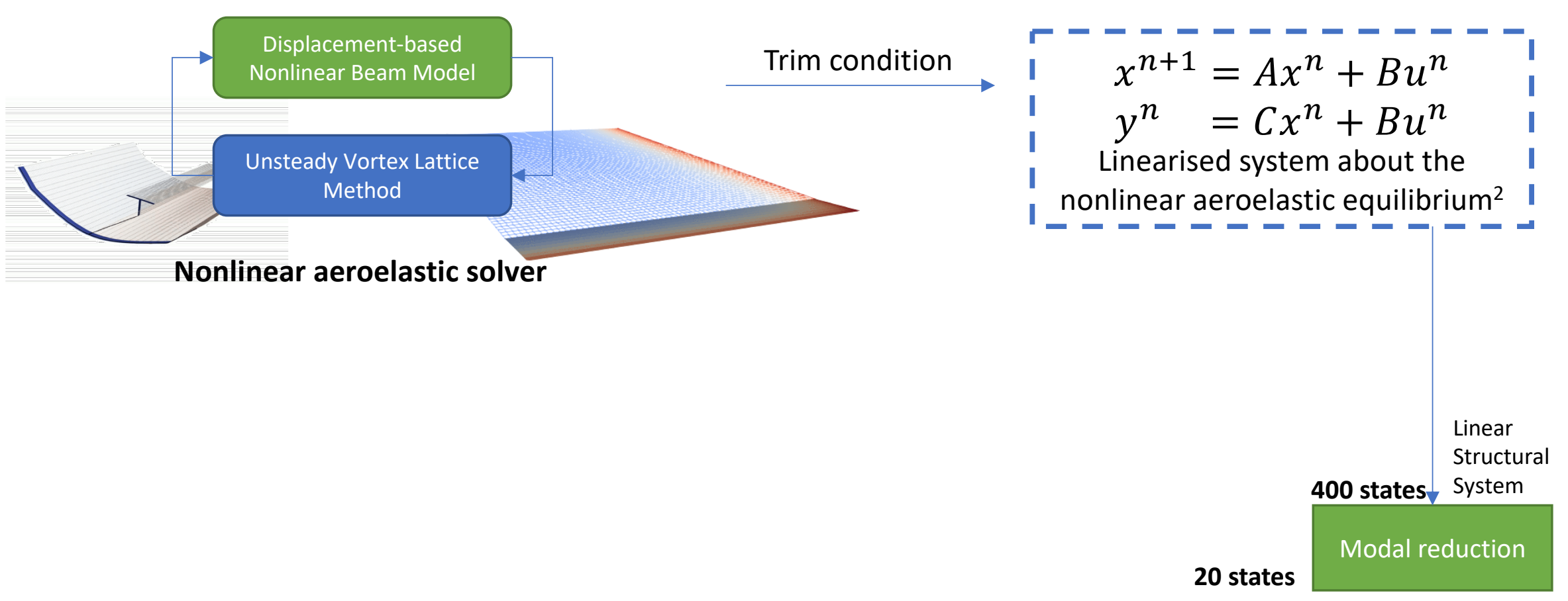




\section{Nonlinear Aeroelastic Modelling}

SHARPy: Simulation of High Aspect Ratio aeroplanes and wind turbines in Python ${ }^{1}$

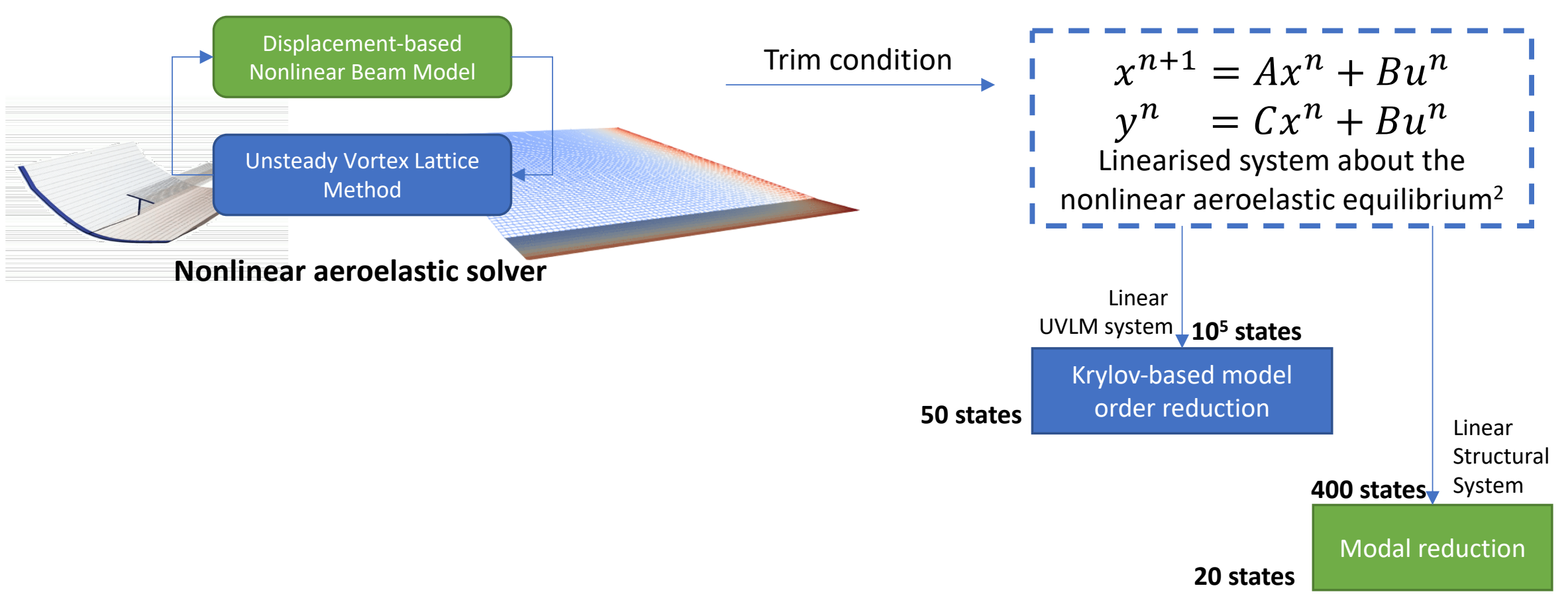




\section{Nonlinear Aeroelastic Modelling}

SHARPy: Simulation of High Aspect Ratio aeroplanes and wind turbines in Python ${ }^{1}$

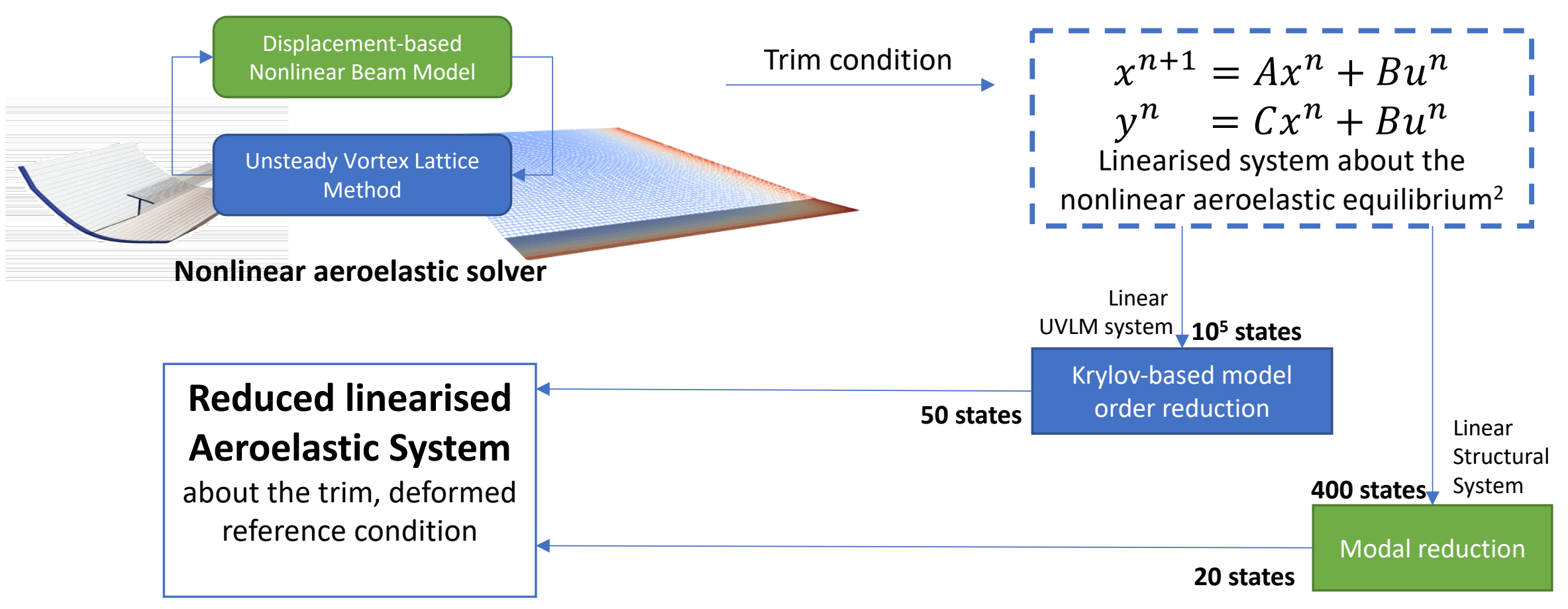




\section{Discrete, Linear Time-Invariant UVLM}

The DLTI UVLM can have thousands of inputs and outputs, depending on the lattice discretisation. Significant penalty on model reduction method, as the order is dependent on the number of transfer functions: 


\section{Discrete, Linear Time-Invariant UVLM}

The DLTI UVLM can have thousands of inputs and outputs, depending on the lattice discretisation. Significant penalty on model reduction method, as the order is dependent on the number of transfer functions:

Project the UVLM onto the structural modal space 


\section{Discrete, Linear Time-Invariant UVLM}

The DLTI UVLM can have thousands of inputs and outputs, depending on the lattice discretisation. Significant penalty on model reduction method, as the order is dependent on the number of transfer functions:

Project the UVLM onto the structural modal space

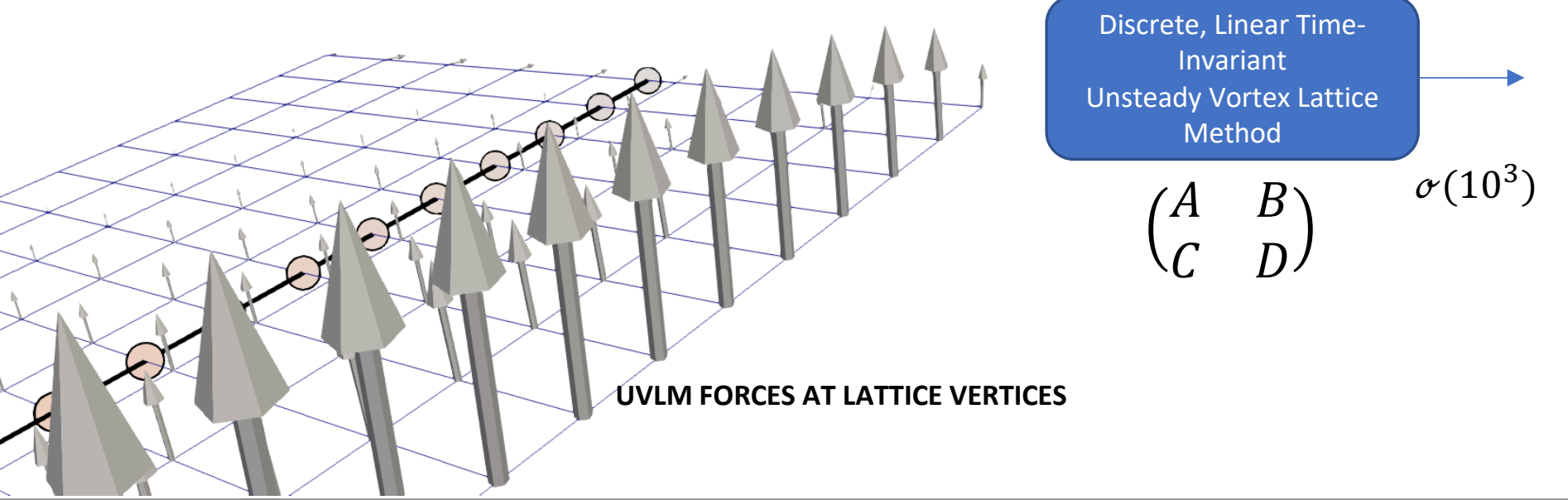




\section{Discrete, Linear Time-Invariant UVLM}

The DLTI UVLM can have thousands of inputs and outputs, depending on the lattice discretisation. Significant penalty on model reduction method, as the order is dependent on the number of transfer functions:

Project the UVLM onto the structural modal space

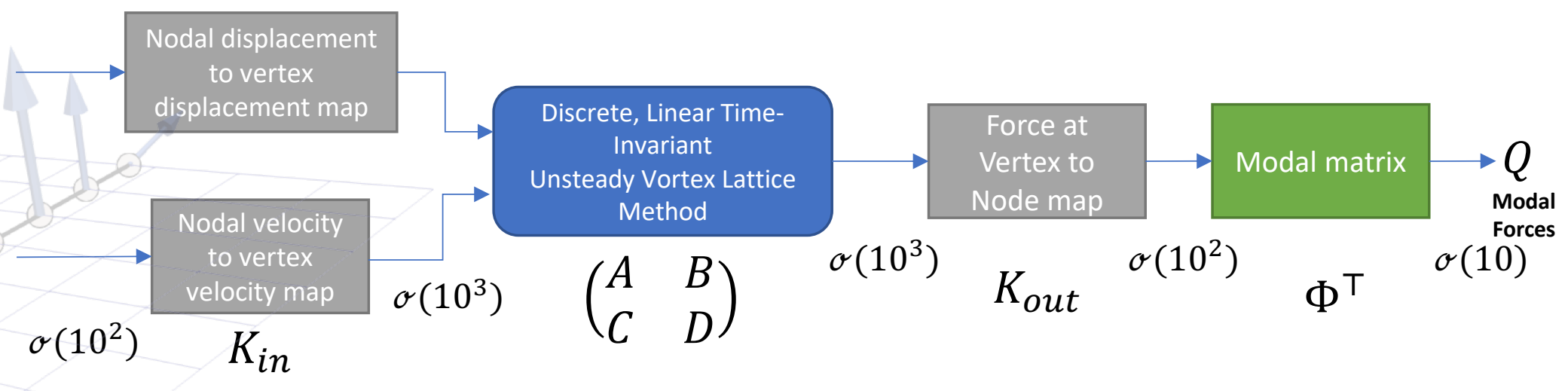




\section{Discrete, Linear Time-Invariant UVLM}

The DLTI UVLM can have thousands of inputs and outputs, depending on the lattice discretisation. Significant penalty on model reduction method, as the order is dependent on the number of transfer functions:

Project the UVLM onto the structural modal space

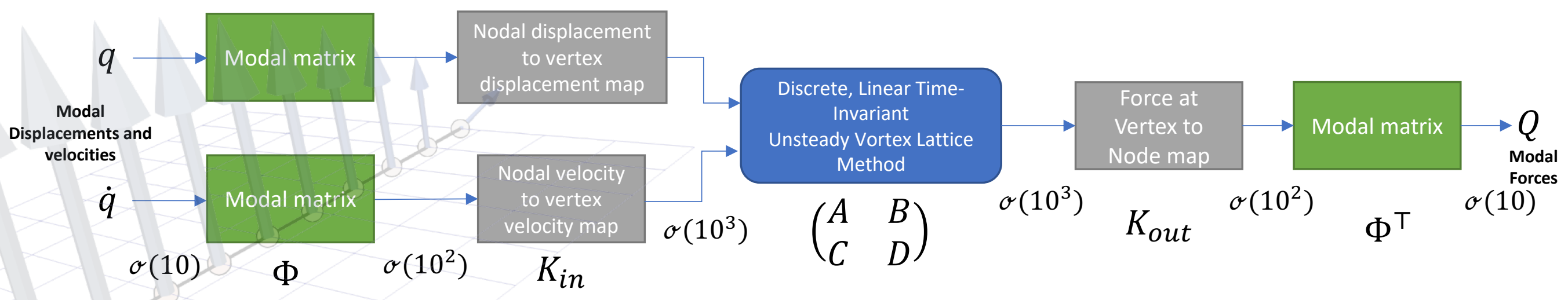




\section{Discrete, Linear Time-Invariant UVLM}

The DLTI UVLM can have thousands of inputs and outputs, depending on the lattice discretisation. Significant penalty on model reduction method, as the order is dependent on the number of transfer functions:

Project the UVLM onto the structural modal space

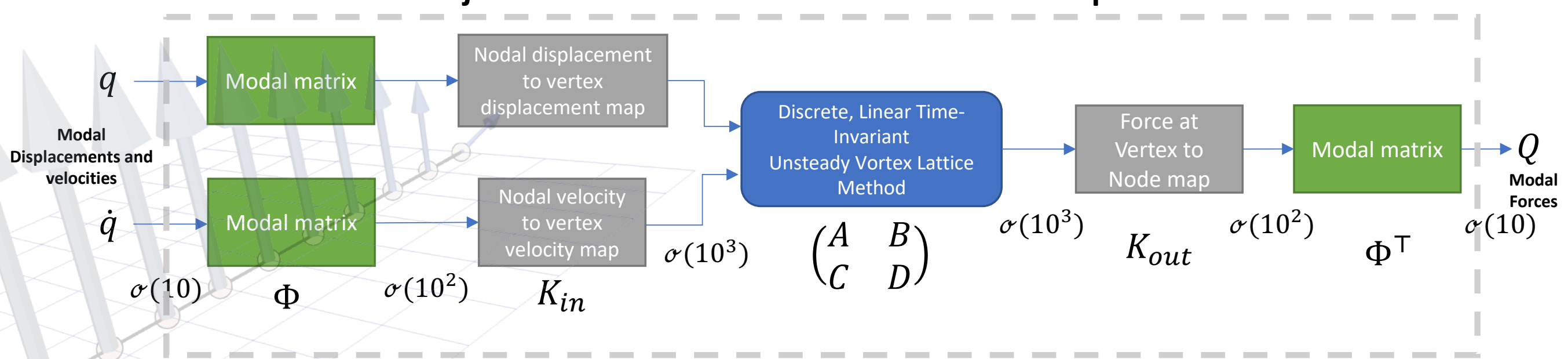

Large state vector, small number of transfer functions $\rightarrow$ suitable for efficient reduction 


\section{Krylov-based model reduction of the DLTI UVLM}

Krylov subspace methods are the chosen model order reduction technique

- Highly suited to deal with large systems

- Can be tailored to frequencies of interest 


\section{Krylov-based model reduction of the DLTI UVLM}

Based on matching the moments of the transfer function

$$
H(s)=C(s I-A)^{-1} B+D \quad \text { Transfer function of a linear, time-invariant system }
$$




\section{Krylov-based model reduction of the DLTI UVLM}

Based on matching the moments of the transfer function

$$
\begin{array}{ll}
H(s)=C(s I-A)^{-1} B+D & \text { Transfer function of a linear, time-invariant system } \\
H(s)=\sum_{j=0}^{\infty} \mu_{\sigma}^{(j)}(\sigma-s)^{j} & \begin{array}{l}
\text { Power series expansion of the transfer function about a } \\
\text { point } \sigma \text { in the complex plane }
\end{array}
\end{array}
$$




\section{Krylov-based model reduction of the DLTI UVLM}

Based on matching the moments of the transfer function

$$
\begin{array}{ll}
H(s)=C(s I-A)^{-1} B+D & \text { Transfer function of a linear, time-invariant system } \\
H(s)=\sum_{j=0}^{\infty j=r} \mu_{\sigma}^{(j)}(\sigma-s)^{j} & \begin{array}{l}
\text { Power series expansion of the transfer function about a } \\
\text { point } \sigma \text { in the complex plane }
\end{array}
\end{array}
$$

The philosophy of the reduction method is to match a small number of derivatives

$$
\mu_{\sigma}^{(j)}=C(\sigma I-A)^{-j-1} B
$$




\section{Krylov-based model reduction of the DLTI UVLM}

Based on matching the moments of the transfer function

$$
\begin{array}{ll}
H(s)=C(s I-A)^{-1} B+D & \text { Transfer function of a linear, time-invariant system } \\
H(s)=\sum_{j=0}^{\infty j=r} \mu_{\sigma}^{(j)}(\sigma-s)^{j} & \begin{array}{l}
\text { Power series expansion of the transfer function about a } \\
\text { point } \sigma \text { in the complex plane }
\end{array}
\end{array}
$$

The philosophy of the reduction method is to match a small number of derivatives

$$
\mu_{\sigma}^{(j)}=C(\sigma I-A)^{-j-1} B
$$

Moment related to the transfer function derivative at the point $\sigma$ in the complex plane

Computing these terms explicitly leads to ill-conditioned matrices and numerical instabilities 


\section{Krylov-based model reduction of the DLTI UVLM}

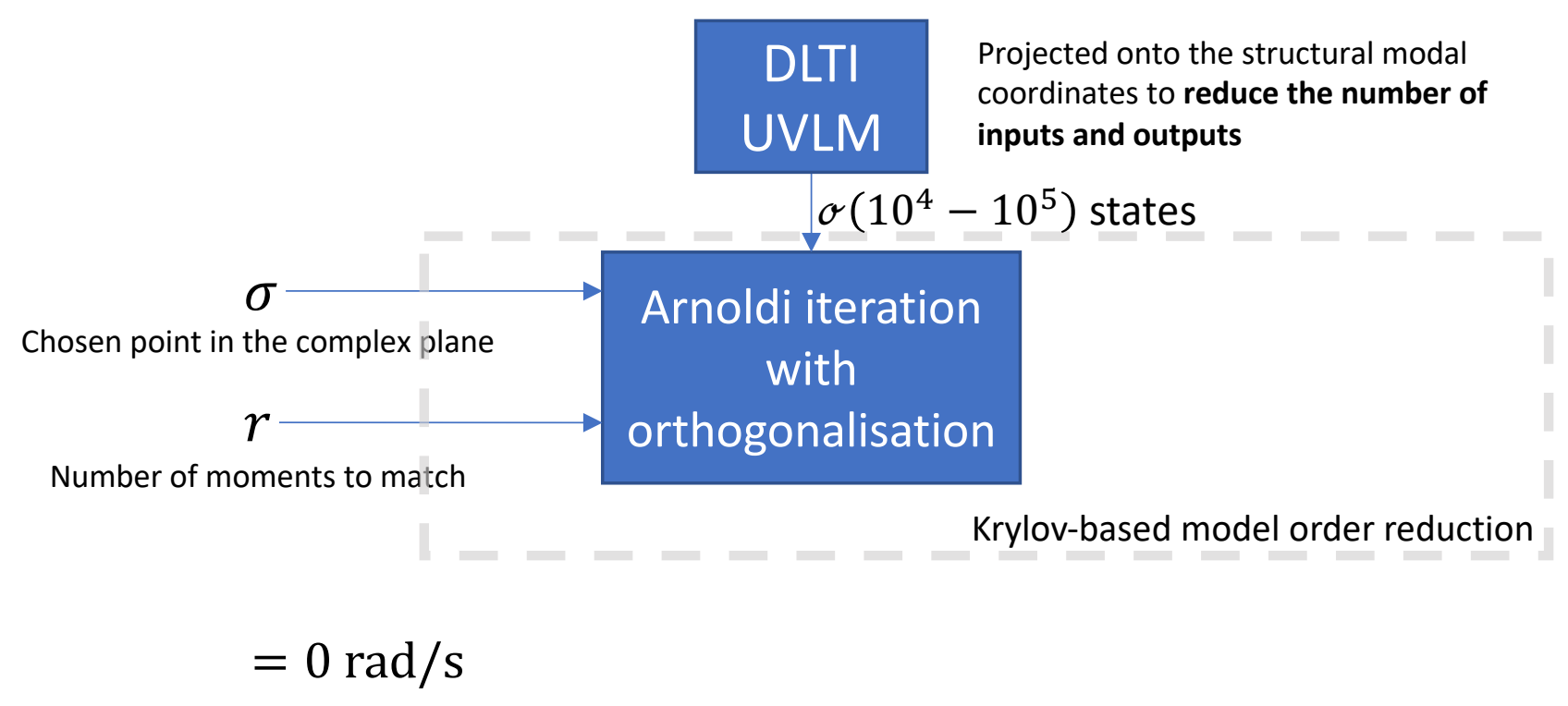

is typically small 


\section{Krylov-based model reduction of the DLTI UVLM}

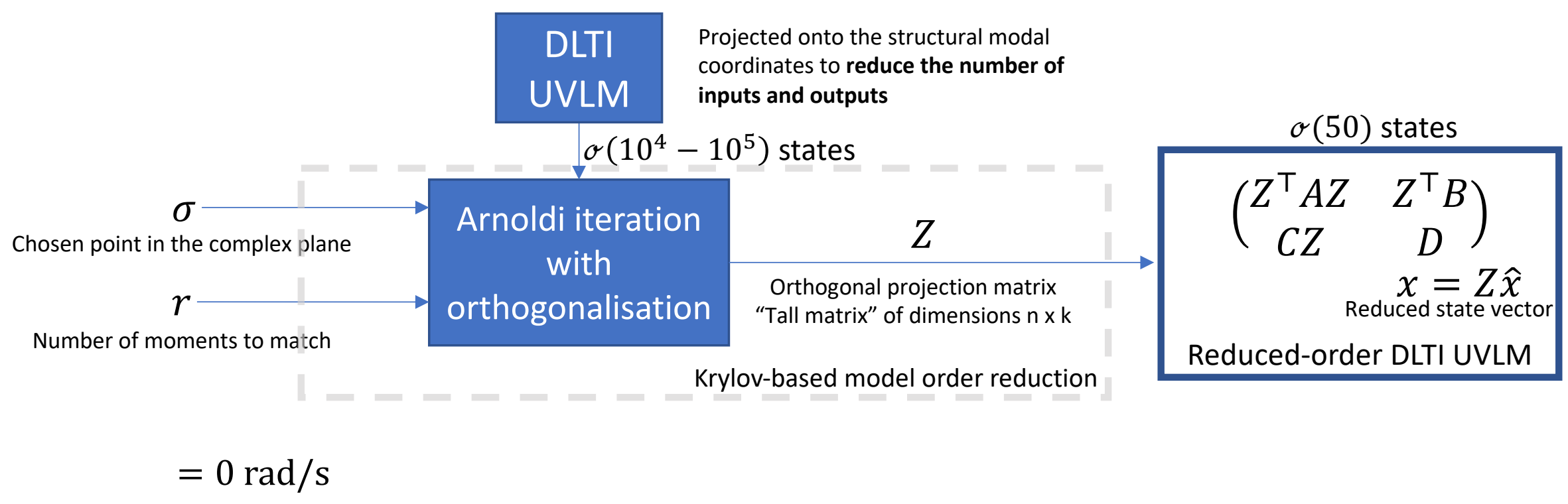

is typically small 


\section{Krylov-based model reduction of the DLTI UVLM}

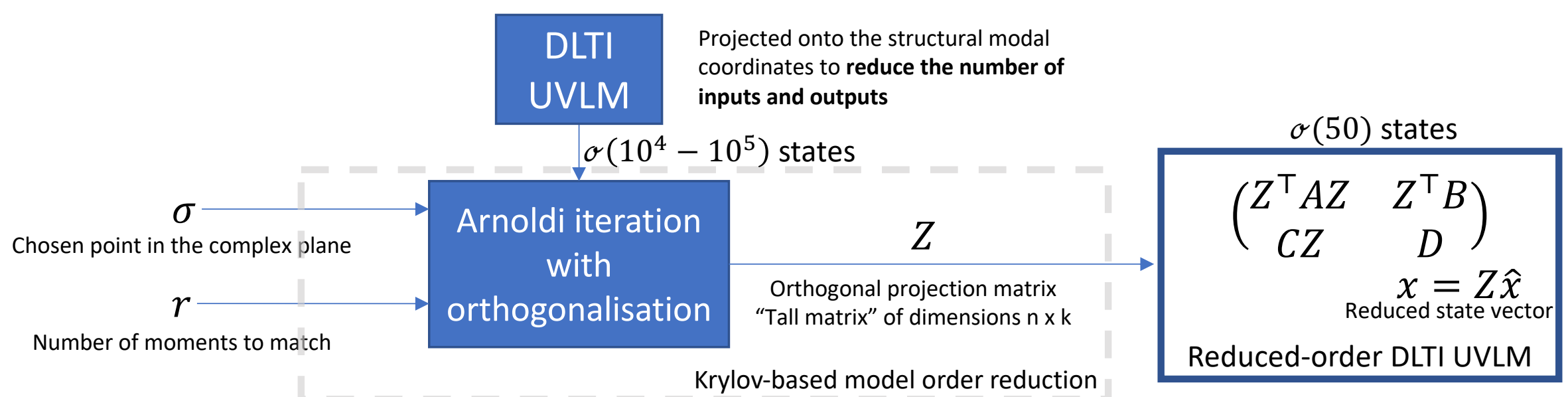

$0 \mathrm{rad} / \mathrm{s}$

Given the UVLM characteristics and interests in the low frequency spectrum: match the steady-state gain $\rightarrow \sigma=0 \mathrm{rad} / \mathrm{s}$ 


\section{Krylov-based model reduction of the DLTI UVLM}

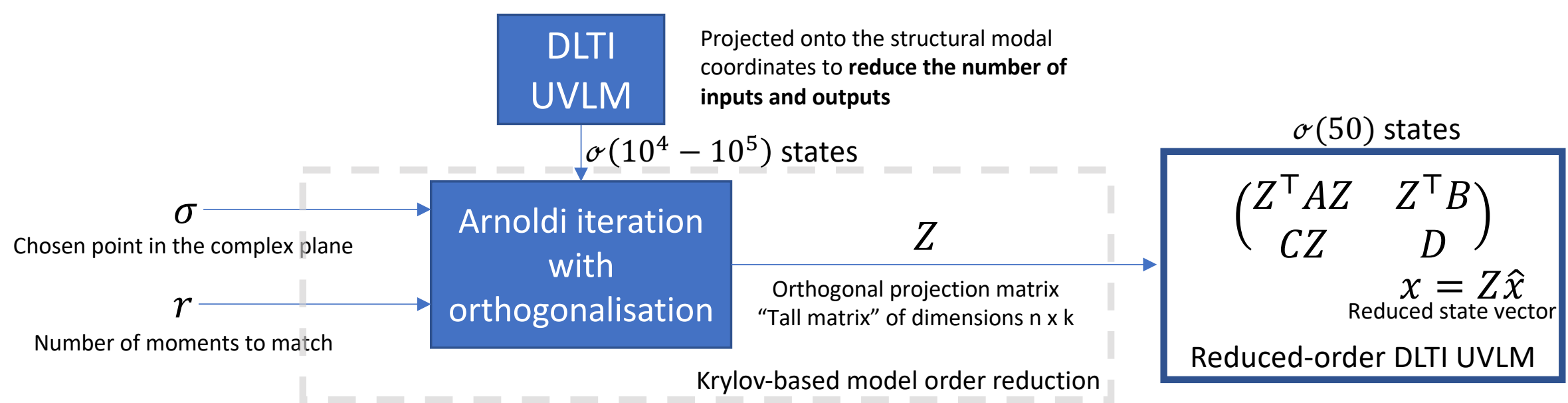

$0 \mathrm{rad} / \mathrm{s}$

Given the UVLM characteristics and interests in the low frequency spectrum: match the steady-state gain $\rightarrow \sigma=0 \mathrm{rad} / \mathrm{s}$

Aerodynamic transfer functions are smooth so $r$ is typically small is typically small 


\section{Krylov-based model reduction of the DLTI UVLM}

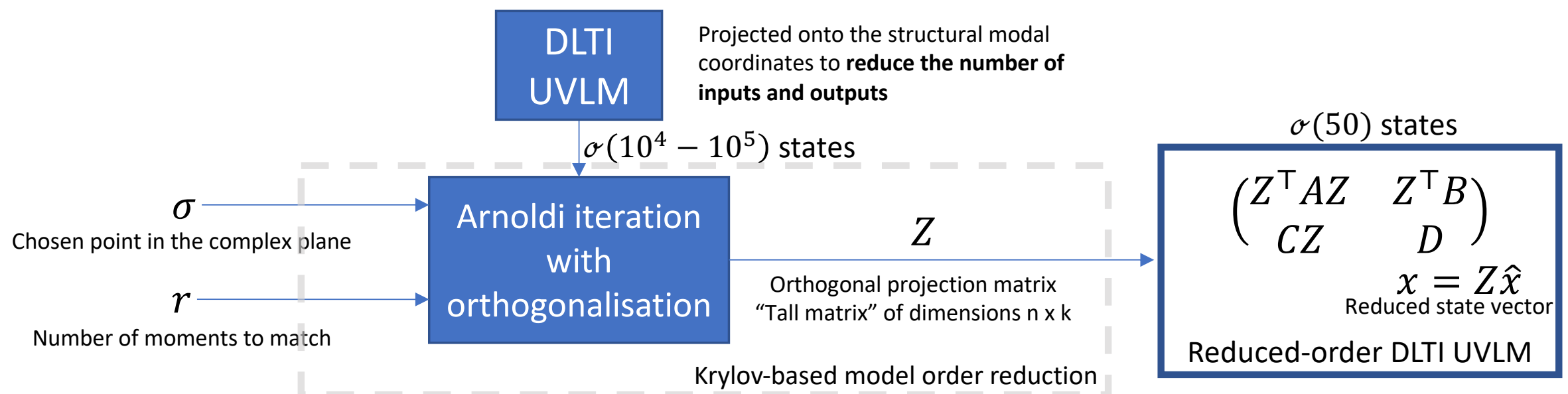

$0 \mathrm{rad} / \mathrm{s}$

Given the UVLM characteristics and interests in the low frequency spectrum: match the steady-state gain $\rightarrow \sigma=0 \mathrm{rad} / \mathrm{s}$

Aerodynamic transfer functions are smooth so $r$ is typically small

is typically small

The order of the ROM is mostly dependent then on the number of inputs and outputs, i.e. the number of structural modes retained 


\section{Library of reduced order aeroelastic models}

Krylov-based reduction of the aerodynamic system

Modal reduction of the structural system 


\section{Library of reduced order aeroelastic models}

Krylov-based reduction of the aerodynamic system

Modal reduction of the structural system

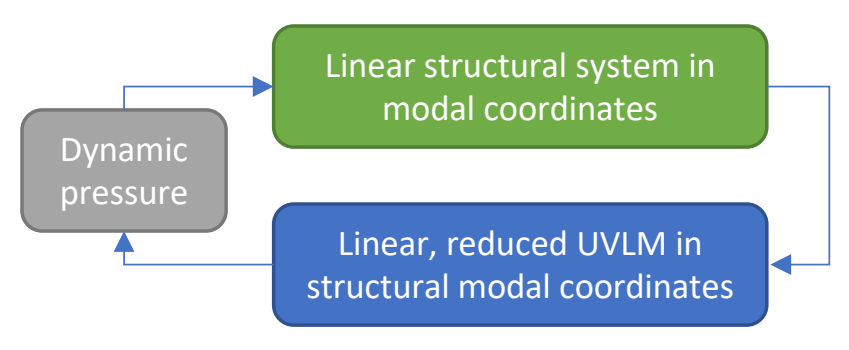




\section{Library of reduced order aeroelastic models}

Krylov-based reduction of the aerodynamic system

Modal reduction of the structural system

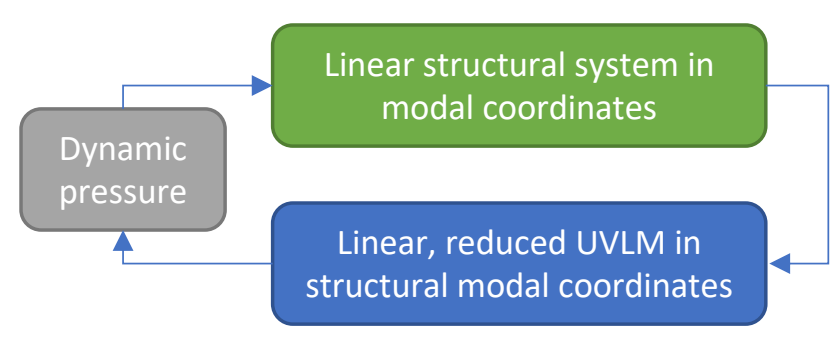




\section{Library of reduced order aeroelastic models}

Krylov-based reduction of the aerodynamic system

Modal reduction of the structural system

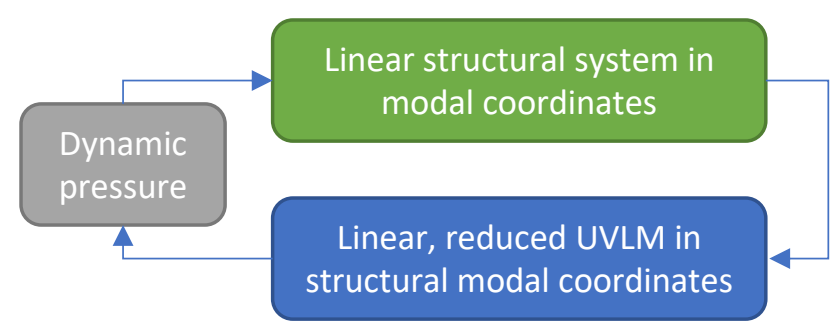

Reduced Linear Aeroelastic System

These can be made a function of a parameter(s) and stored in a library, and we explore two options: 


\section{Library of reduced order aeroelastic models}

Krylov-based reduction of the aerodynamic system

Modal reduction of the structural system

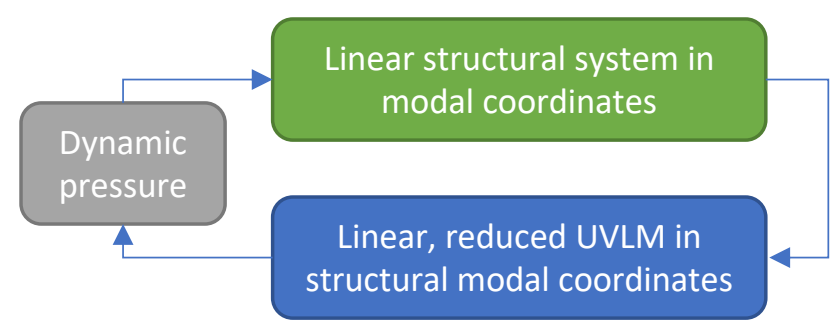

Reduced Linear Aeroelastic System

These can be made a function of a parameter(s) and stored in a library, and we explore two options:

$\Sigma_{\text {aero,1 }}, \Sigma_{\text {aero,2 }} \ldots \Sigma_{\text {aero,K }}$

$\Sigma_{\text {struct }, 1}, \Sigma_{\text {struct, }, \ldots} \ldots \Sigma_{\text {struct, } K}$ 


\section{Library of reduced order aeroelastic models}

Krylov-based reduction of the aerodynamic system

Modal reduction of the structural system

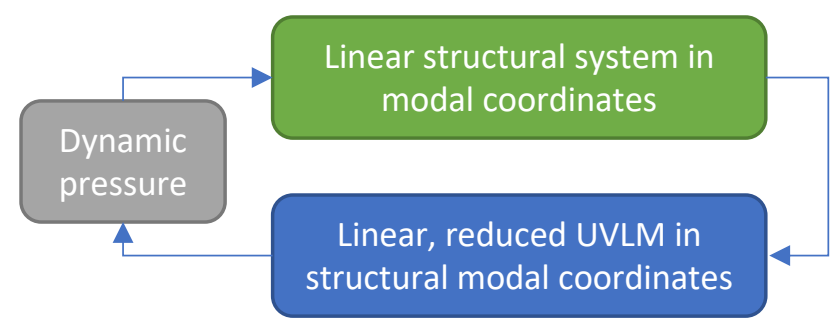

Reduced Linear Aeroelastic System

These can be made a function of a parameter(s) and stored in a library, and we explore two options:
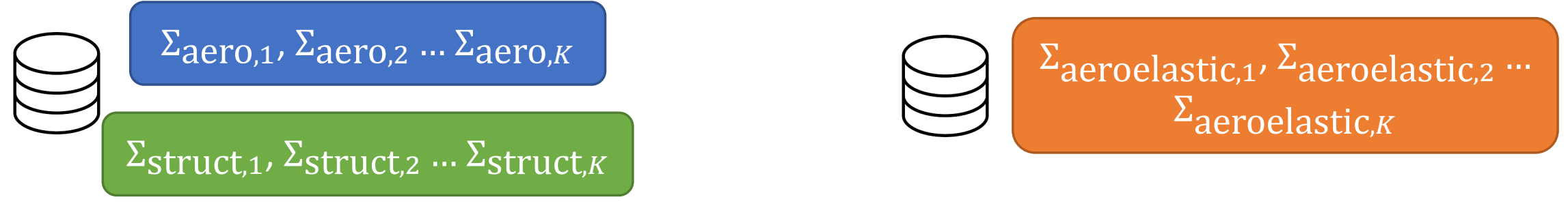


\section{Interpolation of reduced order models}

The interpolation is performed on the system matrices:

$$
A_{\text {new }}=\sum_{i=1}^{N_{K}} w\left(p_{\text {new }}\right) A_{i}
$$

Weighted interpolation of the system matrix to give the interpolated matrix at the new operating condition $p_{\text {new }}$ 


\section{Interpolation of reduced order models}

The interpolation is performed on the system matrices:

$$
A_{\text {new }}=\sum_{i=1}^{N_{K}} w\left(p_{\text {new }}\right) A_{i}
$$

Interpolation cannot be performed directly: the reduced order models have $\Delta$ been obtained independently from one another The reduced state vectors may represent different linear combinations of the Krylov subspaces 


\section{Interpolation of reduced order models}

The interpolation is performed on the system matrices:

$$
A_{\text {new }}=\sum_{i=1}^{N_{K}} w\left(p_{\text {new }}\right) A_{i}
$$

Weighted interpolation of the system matrix to give the interpolated matrix at the new operating condition $p_{\text {new }}$

Interpolation cannot be performed directly: the reduced order models have $\Delta$ been obtained independently from one another

The reduced state vectors may represent different linear combinations of the Krylov subspaces

Transform the reduced states to a common coordinate system $\left(\cdot^{*}\right)$ such that:

$$
x_{1}^{*}=x_{2}^{*}=x_{3}^{*}=\ldots=x_{N_{K}}^{*}
$$

Transformed, reduced order state vectors expressed in a common coordinate system

The transformation is performed by choosing the most relevant dynamics of across all the models in the library ${ }^{3}$ 


\section{Interpolation of reduced order models}

The interpolation is performed on the system matrices:

$$
A_{\text {new }}=\sum_{i=1}^{N_{K}} w\left(p_{\text {new }}\right) A_{i}
$$

Weighted interpolation of the system matrix to give the interpolated matrix at the new operating condition $p_{\text {new }}$

Interpolation cannot be performed directly: the reduced order models have $\Delta$ been obtained independently from one another

The reduced state vectors may represent different linear combinations of the Krylov subspaces

Transform the reduced states to a common coordinate system $\left(\cdot^{*}\right)$ such that:

$$
x_{1}^{*}=x_{2}^{*}=x_{3}^{*}=\ldots=x_{N_{K}}^{*}
$$

Transformed, reduced order state vectors expressed in a common coordinate system

The transformation is performed by choosing the most relevant dynamics of across all the models in the library ${ }^{3}$ 


\section{Case Study: Horten Wing}

Flexible, flying-wing configuration ${ }^{4}$

High aspect ratio, span-loaded aircraft that suffers from Body Freedom Flutter

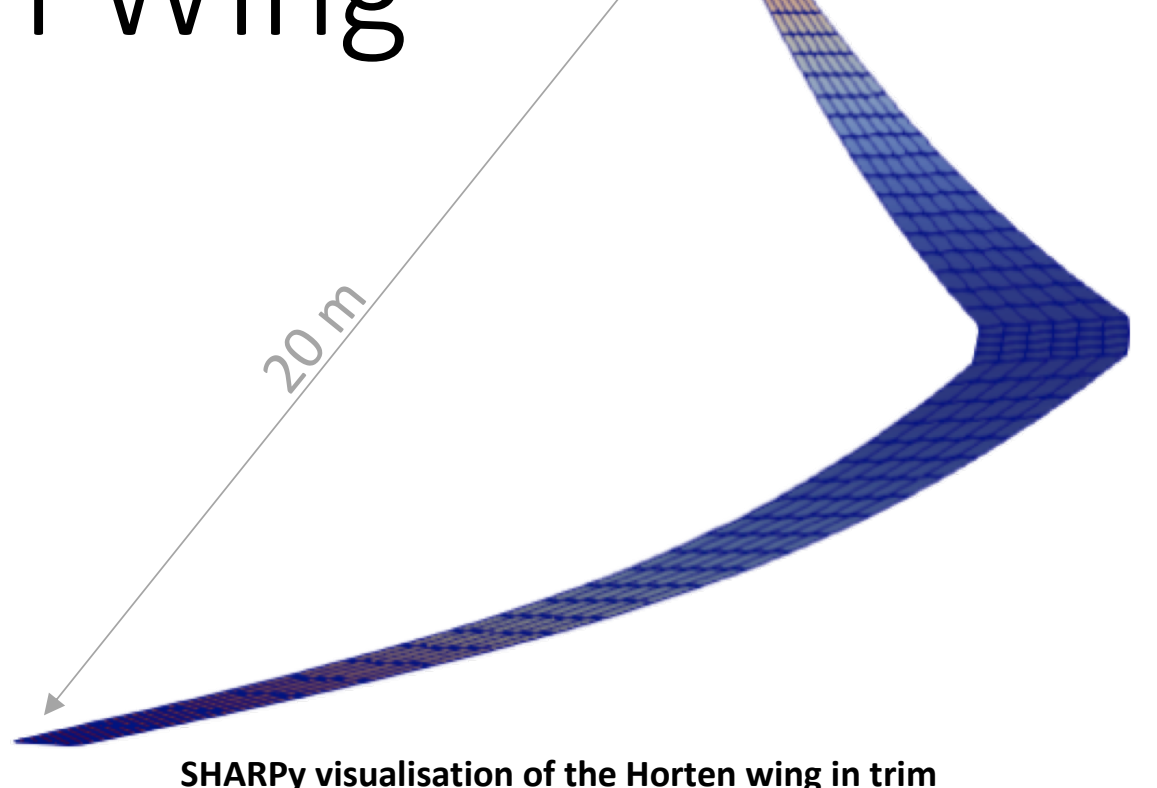




\section{Case Study: Horten Wing}

Flexible, flying-wing configuration ${ }^{4}$

High aspect ratio, span-loaded aircraft that suffers from Body Freedom Flutter

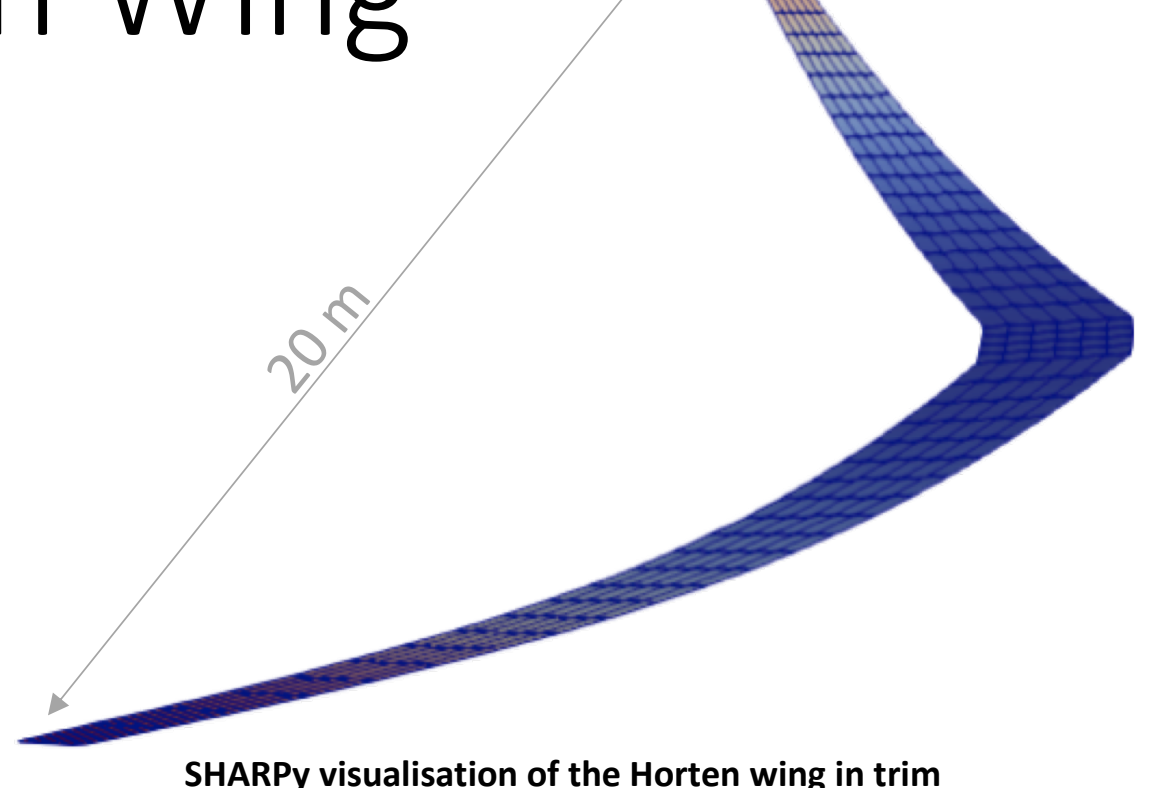

Evaluate the performance of the Krylov reduction and use the parametric interpolation to predict the onset of body freedom flutter 


\section{Krylov Reduction of Horten Aerodynamic} System 


\section{Krylov Reduction of Horten Aerodynamic} System

Trim the aircraft (nonlinear aeroelastic equilibrium) 


\section{Krylov Reduction of Horten Aerodynamic System}

Trim the aircraft (nonlinear aeroelastic equilibrium)

Linearise the UVLM system 


\section{Krylov Reduction of Horten Aerodynamic System}

Trim the aircraft (nonlinear aeroelastic equilibrium)

Linearise the UVLM system

Project the linearised UVLM onto the rigid-body modes and 11 flexible modes 


\section{Krylov Reduction of Horten Aerodynamic System}

Trim the aircraft (nonlinear aeroelastic equilibrium)

Linearise the UVLM system

Project the linearised UVLM onto the rigid-body modes and 11 flexible modes

Perform the Krylov subspace reduction about the steady-state gain modifying the number of moments matched, from 1 to 10 


\section{Krylov Reduction of Horten Aerodynamic}

\section{System}

Trim the aircraft (nonlinear aeroelastic equilibrium)

Linearise the UVLM system

Project the linearised UVLM onto the rigid-body modes and 11 flexible modes

Perform the Krylov subspace reduction about the steady-state gain modifying the number of moments matched, from 1 to 10

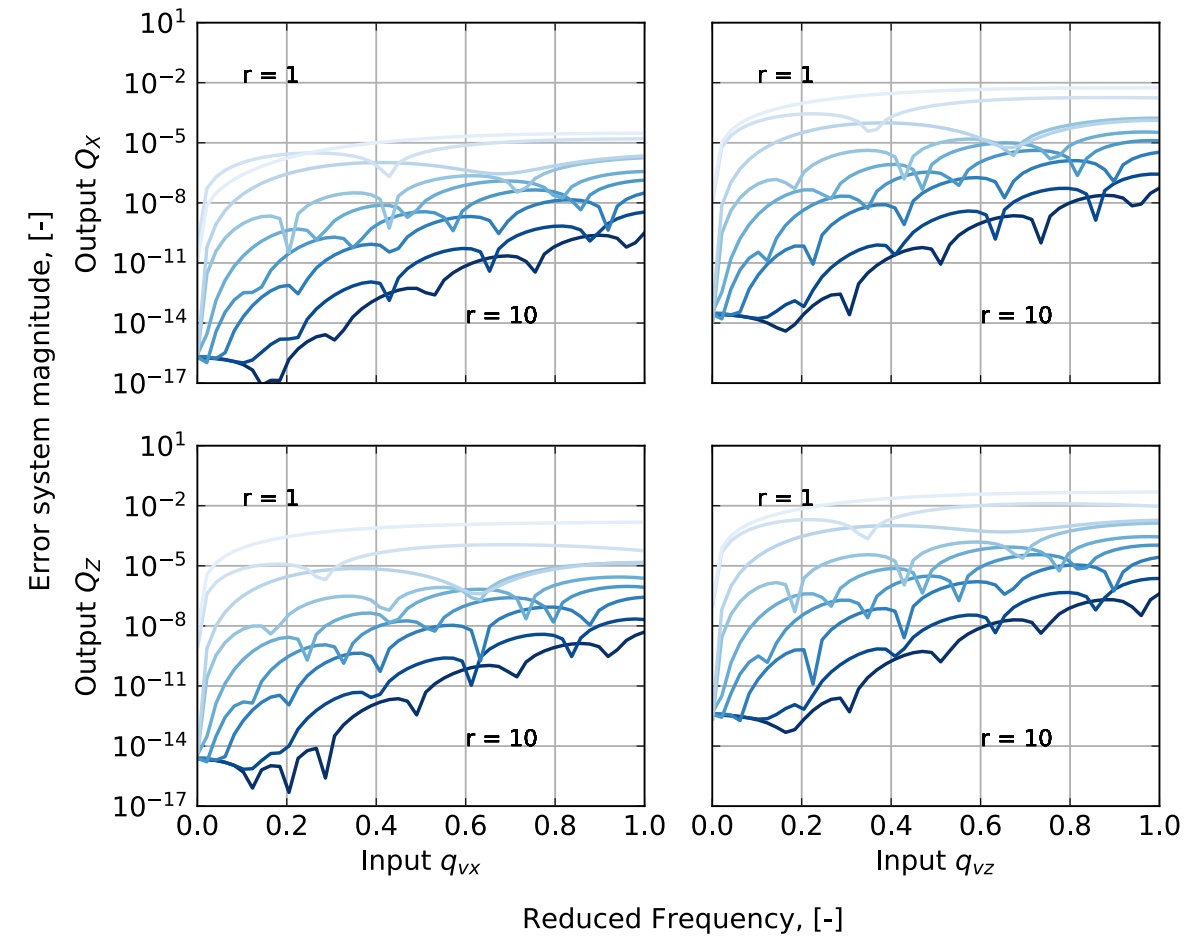

Transfer functions of the error system. Horizontal and Vertical velocity inputs to Horizontal and Vertical Force outputs 


\section{Krylov Reduction of Horten Aerodynamic}

\section{System}

Trim the aircraft (nonlinear aeroelastic equilibrium)

Linearise the UVLM system

Project the linearised UVLM onto the rigid-body modes and 11 flexible modes

Perform the Krylov subspace reduction about the steady-state gain modifying the number of moments matched, from 1 to 10

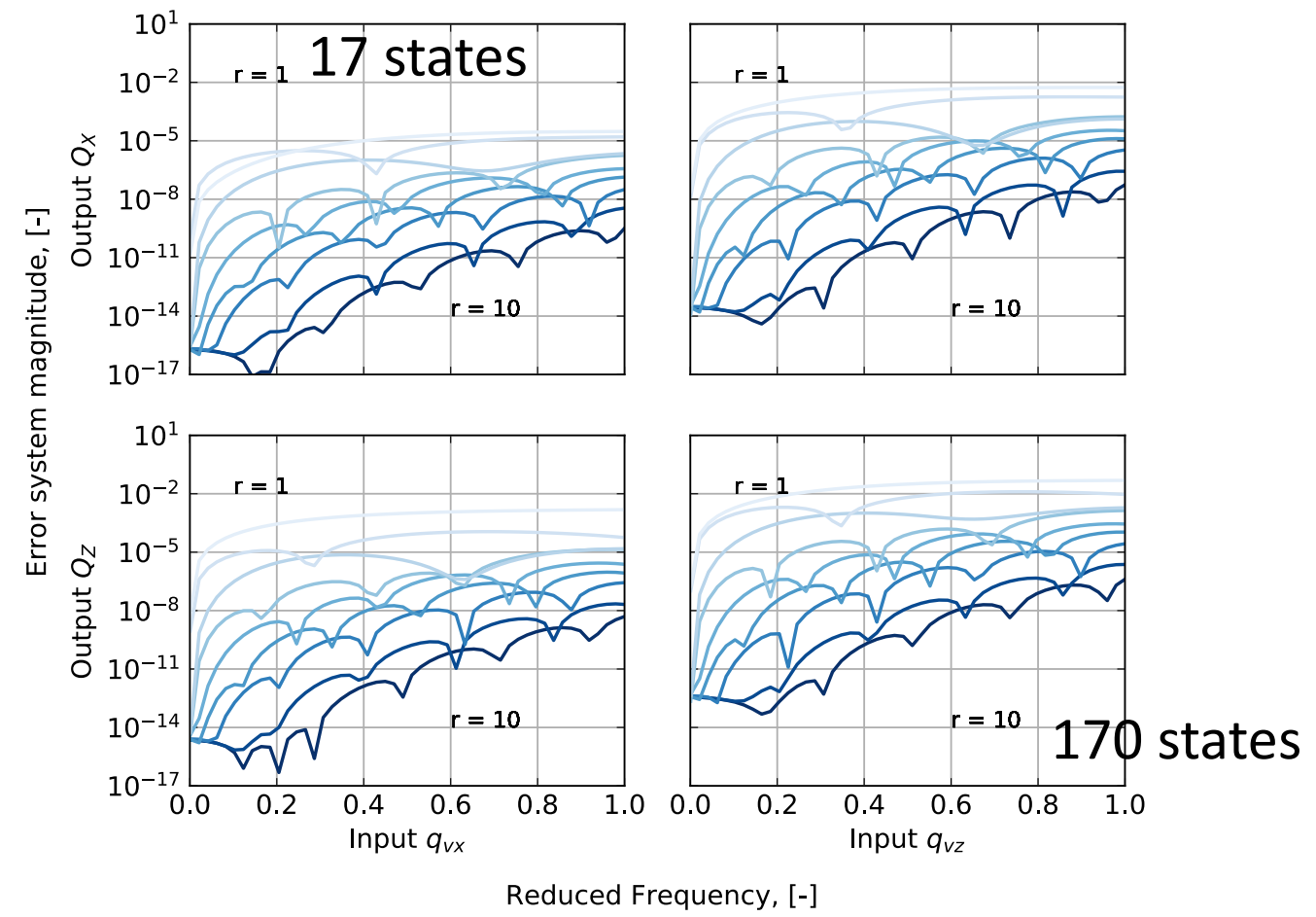

Transfer functions of the error system. Horizontal and Vertical velocity inputs to Horizontal and Vertical Force outputs 


\section{Properties of interpolated models}

Sample the flight envelope:

Single parameter, flight velocity

Trim $\rightarrow$ Linearise $\rightarrow$ Reduce (17 modes, 6 moments, error $1 \mathrm{e}-6$ at $\mathrm{k}=0.3$ )

$\rightarrow$ Store in library.

$\rightarrow$ Transform and interpolate for the desired velocity 


\section{Stability properties of interpolated models}

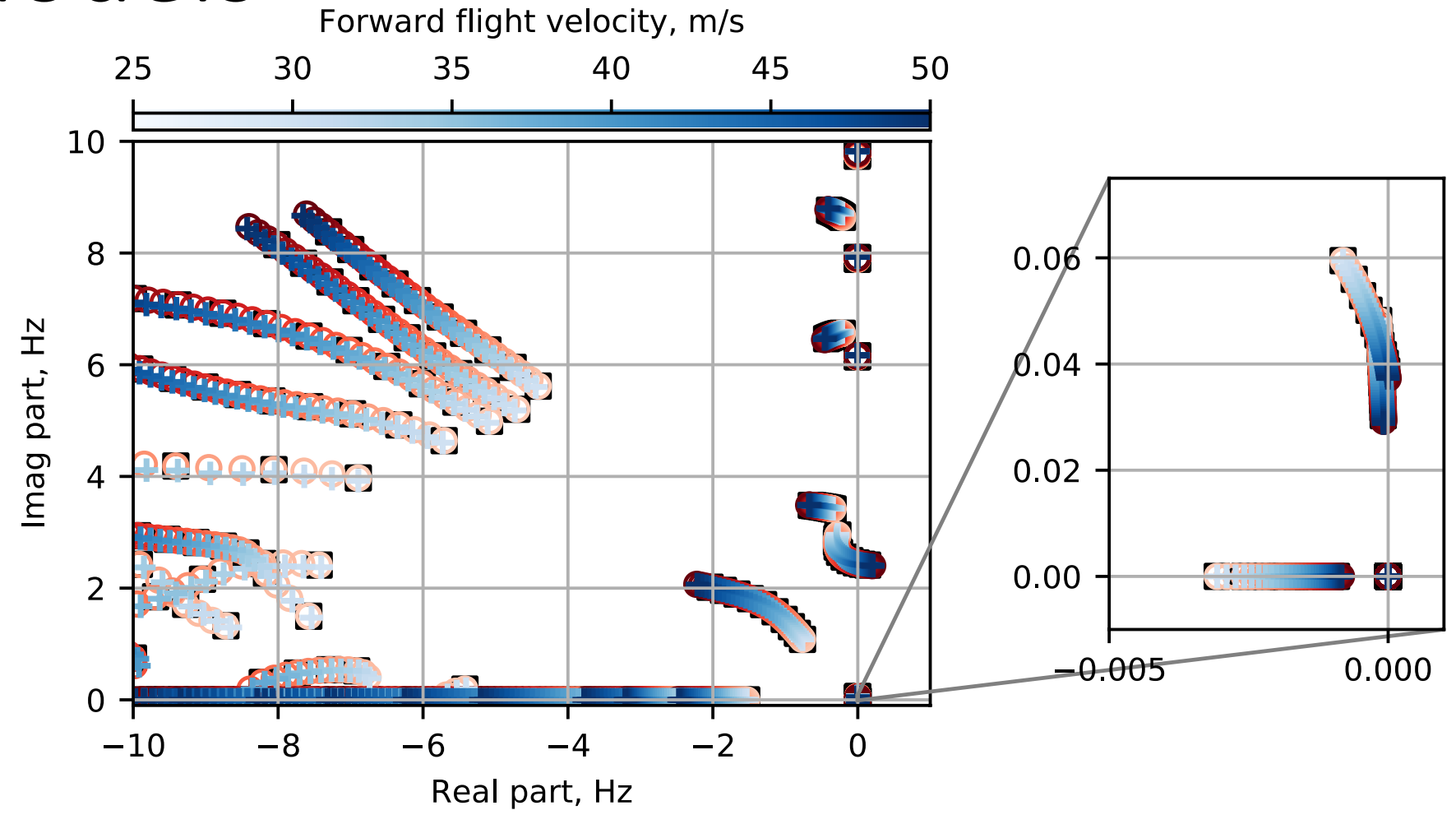




\section{Stability properties of interpolated} models

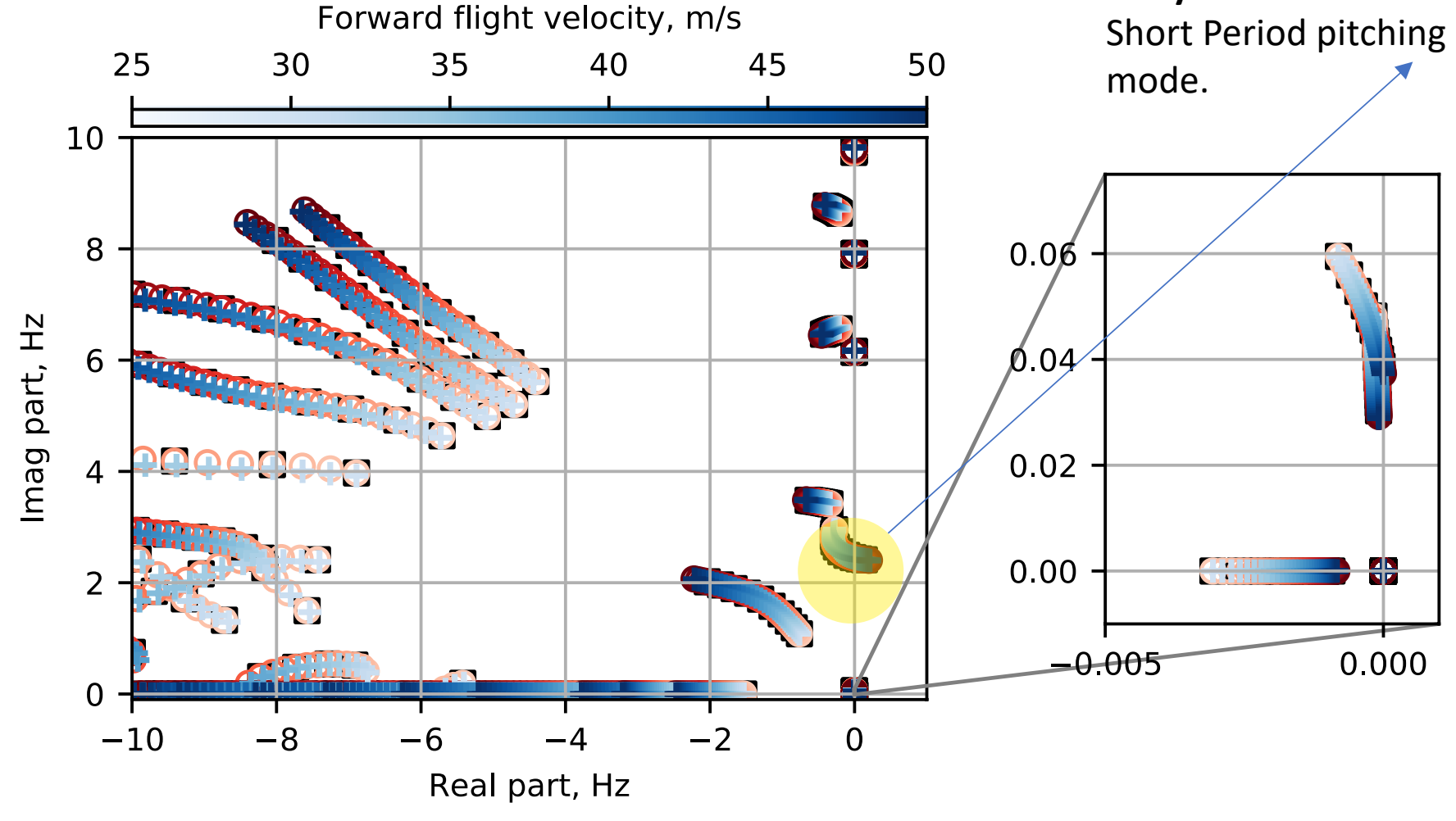

Body Freedom Flutter instability, $1^{\text {st }}$ bending + Short Period pitching oscillations. "Pecking-like"

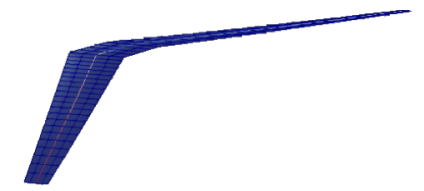

+ Interpolated data

O Actual data

$\square$ Source data 


\section{Concluding remarks}

Offline phase: Efficient model order reduction of multi-input multioutput systems using Krylov subspaces.

Online phase: leverage on reduced matrices to quickly interpolate the system for new operating conditions

Preliminary demonstrator on a free flying aircraft of the capabilities of interpolated, parametric models

This computes online an appropriate model for the current flight condition, paving the way to real time use for control applications. 


\section{Parametric Krylov-based order reduction of aircraft aeroelastic models} Computational Reduced-Order Models I 


\section{References}

[1] Del Carre, A., Muñoz-Simón, A., Goizueta, N., and Palacios, R., "SHARPy: A dynamic aeroelastic simulation toolbox for very flexible aircraft and wind turbines," Journal of Open Source Software, Vol. 4, No. 44, 2019, p. 1885.

[2] Maraniello, S., and Palacios, R., "State-Space Realizations and Internal Balancing in Potential-Flow Aerodynamics with Arbitrary Kinematics," AIAA Journal, Vol. 57, No. 6, 2019.

[3] Panzer, H., Mohring, J., Eid, R., and Lohmann, B., "Parametrische ordnungsreduktion mittels matrixinterpolation," At Automatisierungstechnik, Vol. 58, No. 8, 2010, pp. 475484. https://doi.org/10.1524/auto.2010.0863.

[4] Richards, P. W., Yao, Y., Herd, R. A., Hodges, D. H., and Mardanpour, P., "Effect of Inertial and Constitutive Properties on Body-Freedom Flutter for Flying Wings," Journal of Aircraft, 2016. https://doi.org/10.2514/1.C033435. 